\title{
CP Violation in the scalar sector
}

\section{Emmanuel-Costa}

Centro de Física Teórica de Partículas - CFTP

Instituto Superior Técnico - IST, Universidade de Lisboa, Av. Rovisco Pais, P-1049-001 Lisboa, Portugal

E-mail: david.costa@tecnico.ulisboa.pt

\section{O. M. Ogreid}

Bergen University College, Bergen, Norway

E-mail: omoahib.no

\section{P. Osland*}

University of Bergen, Norway

E-mail: per.oslandeift.uib.no

\section{N. Rebelo}

Centro de Física Teórica de Partículas - CFTP and Departamento de Física Instituto Superior Técnico - IST, Universidade de Lisboa, Av. Rovisco Pais,

P-1049-001 Lisboa, Portugal

E-mail: rebelo@tecnico.ulisboa.pt

Models with an extended scalar sector may in principle provide new sources of CP violation originating in the scalar potential. One of the simplest ways to implement this idea is to have $\mathrm{CP}$ violation in a two-Higgs-doublet model. Here, it leads to CP violation in trilinear weak gauge boson couplings. We discuss how these couplings are becoming constrained in the alignment limit. In a model with three Higgs doublets, subject to an $S_{3}$ symmetry, several complex vacua are possible. Some of these, but not all, may lead to spontaneous CP violation.

Proceedings of the Corfu Summer Institute 2015 "School and Workshops on Elementary Particle Physics and Gravity"

1-27 September 2015

Corfu, Greece

${ }^{*}$ Speaker. 


\section{Introduction}

In our attempts to identify physics beyond the standard model, one may seek guidance from the fact that baryogenesis [1] requires additional CP violation. Actually, it has long been known that the extension of the standard model with an extra $\mathrm{SU}(2) \times \mathrm{U}(1)$ scalar doublet introduces additional sources of $\mathrm{CP}$ violation. In fact, models with two Higgs doublets can violate $\mathrm{CP}$ either explicitly or spontaneously. Spontaneous CP violation [2] has the attractive feature of putting on an equal footing $\mathrm{CP}$ and electroweak symmetry breaking. It should be stressed that spontaneous $\mathrm{CP}$ violation can only occur, provided the Lagrangian conserves $\mathrm{CP}$.

We shall here briefly review the constraints on CP violation in the Two-Higgs-Doublet Model (2HDM) and then discuss a scalar potential with three Higgs doublets. The general three-Higgsdoublet model has many parameters, so we will restrict ourselves to the ten-parameter $S_{3}$-symmetric potential. The additional, discrete, symmetry may also provide a framework for dark matter.

The analysis presented here is important for model building. Symmetries have the important feature of reducing the number of free parameters and at the same time leading to predictions that can in principle be verified experimentally at the LHC.

\section{CP violation in the $2 \mathrm{HDM}$}

One of the simplest models that allows for CP violation in the scalar sector is the Two-HiggsDoublet Model (2HDM). When the three neutral Higgs fields of this model mix, CP will be violated, either explicitly or spontaneously [2]. This mixing, which yields the three states $H_{1}, H_{2}$ and $H_{3}$, can be described by two additional mixing angles, replacing the familiar mixing angle $\alpha$ by a set of three, $\left(\alpha_{1}, \alpha_{2}, \alpha_{3}\right)$ [3]. The regions in this parameter space where explicit and spontaneous CP violation can take place, have been discussed in Ref. [4]. In fact, internal consistency and experimental constraints allow some amount of mixing, see, for example, Refs. [5-8].

From one point of view, $\mathrm{CP}$ violation is attractive since it may make baryogenesis possible, from another, it offers CP-violating observables [9-11] that one could try to measure or constrain experimentally. When neither of the three neutral Higgs bosons is an eigenstate of CP, then the neutral gauge boson will have trilinear couplings with all three pairs of neutral scalars. All these will also have trilinear coupings with the charged pair.

Conditions for a two-Higgs-doublet potential to conserve $\mathrm{CP}$ at the Lagrangian level expressed in terms of Higgs basis invariants and which are independent of the vacuum expectation values were presented in Refs. [12] and [13]. Within the bosonic sector of the 2HDM, i.e., without specifying the Yukawa couplings, CP-violating observables can all be expressed in terms of three invariants, $\operatorname{Im} J_{1}, \operatorname{Im} J_{2}$ and $\operatorname{Im} J_{30}$. These may in turn be expressed by the masses of the neutral sector, $\left(M_{1}, M_{2}, M_{3}\right)$, as well as six couplings, in Ref. [11] denoted $e_{i}$ and $q_{i}, i=1,2,3$. Here, $e_{i}$ parametrizes the $Z Z H_{i}$ coupling strength, as well as the $Z H_{j} H_{k}$ coupling (for $i \neq j \neq k \neq i$ ), whereas the $q_{i}$ parametrizes the $H^{+} H^{-} H_{i}$ coupling. The quantity $\operatorname{Im} J_{2}$ actually induces interesting CP-violating effects in effective $Z Z Z$ and $Z W W$ vertices [14-16].

While the data allow some amount of mixing, recent data on the $125 \mathrm{GeV}$ Higgs particle (assumed to be the lightest one, $H_{1}$ ) actually point to the decoupling limit [17], in which its couplings 
to the gauge bosons coincide with those of the Standard Model. In particular, this implies

$$
e_{1}=v, \quad e_{2}=0, \quad e_{3}=0
$$

where $v=246 \mathrm{GeV}$. These values imply $[11,18]$

$$
\begin{aligned}
\operatorname{Im} J_{1} & =0, \\
\operatorname{Im} J_{2} & =0, \\
\operatorname{Im} J_{30} & =\frac{q_{2} q_{3}}{v^{4}}\left(M_{3}^{2}-M_{2}^{2}\right) .
\end{aligned}
$$

Recalling that $q_{2}$ and $q_{3}$ refer to the coupling strengths of a charged Higgs pair to the two heavier neutral ones, it is clear that it would be very challenging to try to measure the $\mathrm{CP}$-violating quantity $\operatorname{Im} J_{30}$.

\section{CP violation in the $\mathrm{S}_{3}$-symmetric 3-Higgs-doublet model}

The $S_{3}$-symmetric three-Higgs-doublet potential, which is defined in terms of ten parameters, has a very rich structure. In the irreducible-representation framework (IRF), where the $S U(2) \times$ $U(1)$ doublets $h_{1}$ and $h_{2}$ form an $S_{3}$ doublet, whereas $h_{S}$ is an $S_{3}$ singlet, it can be written as [19]

$$
\begin{aligned}
V & =\mu_{0}^{2} h_{S}^{\dagger} h_{S}+\mu_{1}^{2}\left(h_{1}^{\dagger} h_{1}+h_{2}^{\dagger} h_{2}\right) \\
& +\lambda_{1}\left(h_{1}^{\dagger} h_{1}+h_{2}^{\dagger} h_{2}\right)^{2}+\lambda_{2}\left(h_{1}^{\dagger} h_{2}-h_{2}^{\dagger} h_{1}\right)^{2}+\lambda_{3}\left[\left(h_{1}^{\dagger} h_{1}-h_{2}^{\dagger} h_{2}\right)^{2}+\left(h_{1}^{\dagger} h_{2}+h_{2}^{\dagger} h_{1}\right)^{2}\right] \\
& +\lambda_{4}\left[\left(h_{S}^{\dagger} h_{1}\right)\left(h_{1}^{\dagger} h_{2}+h_{2}^{\dagger} h_{1}\right)+\left(h_{S}^{\dagger} h_{2}\right)\left(h_{1}^{\dagger} h_{1}-h_{2}^{\dagger} h_{2}\right)+\text { h.c. }\right]+\lambda_{5}\left(h_{S}^{\dagger} h_{S}\right)\left(h_{1}^{\dagger} h_{1}+h_{2}^{\dagger} h_{2}\right) \\
& +\lambda_{6}\left[\left(h_{S}^{\dagger} h_{1}\right)\left(h_{1}^{\dagger} h_{S}\right)+\left(h_{S}^{\dagger} h_{2}\right)\left(h_{2}^{\dagger} h_{S}\right)\right]+\lambda_{7}\left[\left(h_{S}^{\dagger} h_{1}\right)\left(h_{S}^{\dagger} h_{1}\right)+\left(h_{S}^{\dagger} h_{2}\right)\left(h_{S}^{\dagger} h_{2}\right)+\text { h.c. }\right] \\
& +\lambda_{8}\left(h_{S}^{\dagger} h_{S}\right)^{2} .
\end{aligned}
$$

The same ten-parameter potential can also be represented in a complementary, reducible-representation framework (RRF), where the three $S U(2) \times U(1)$ doublets $\left(\phi_{1}, \phi_{2}, \phi_{3}\right)$ are treated on an equal footing [20]. There is a linear mapping between these two potentials, as physical models they are thus equivalent until some other sector is specified, like for example Yukawa couplings.

A couple of features of this potential are worth stressing:

- The potential is invariant under $h_{1} \rightarrow-h_{1}$, but not under $h_{2} \rightarrow-h_{2}$.

- For $\lambda_{4}=0$, the potential has an additional SO(2) symmetry. In addition, the potential is then invariant under $h_{2} \rightarrow-h_{2}$, and under $h_{1} \leftrightarrow h_{2}$.

Recently, a complete catalogue of possible vacua has been given [21], with an emphasis on the complex ones and the corresponding constraints on the parameters of the potential. We list the complex vacua in Table 1. The IRF specification, in terms of vacuum expectation values (vevs) $\left(w_{1}, w_{2}, w_{S}\right)$ is given for all of them, whereas the corresponding RRF specification in terms of the vevs $\left(\rho_{1}, \rho_{2}, \rho_{3}\right)$ is only given for the simpler cases. The minima are determined by solving minimization conditions for three moduli and two relative phases, a total of five conditions. For most of the vacua, these five conditions are not independent. Indeed, the roman numeral making 
Table 1: Complex vacua (after Ref. [21]). Symbols with a "hat" (like $\hat{w}_{S}$ ) are real and positive. The vacua labelled with a checkmark $(\checkmark)$ violate CP spontaneously, whereas those labelled with an asterisk $\left({ }^{*}\right)$ are in fact real, due to the constraints that have to be imposed.

\begin{tabular}{|c|c|c|c|}
\hline Name & IIRF (Irreducible Rep.) & RRF (Reducible Rep.) & SCPV \\
\hline & $w_{1}, w_{2}, w_{S}$ & $\rho_{1}, \rho_{2}, \rho_{3}$ & \\
\hline C-I-a & $\hat{w}_{1}, \pm i \hat{w}_{1}, 0$ & $x, x e^{ \pm \frac{2 \pi i}{3}}, x e^{\mp \frac{2 \pi i}{3}}$ & \\
\hline C-III-a & $0, \hat{w}_{2} e^{i \sigma_{2}}, \hat{w}_{S}$ & $y, y, x e^{i \tau}$ & $\overline{\bar{\checkmark}}$ \\
\hline C-III-b & $\pm i \hat{w}_{1}, 0, \hat{w}_{S}$ & $x+i y, x-i y, x$ & \\
\hline C-III-c & $\hat{w}_{1} e^{i \sigma_{1}}, \hat{w}_{2} e^{i \sigma_{2}}, 0$ & & $\checkmark$ \\
\hline C-III-d & $\pm i \hat{w}_{1}, \hat{w}_{2}, \hat{w}_{S}$ & $x e^{i \tau}, x e^{-i \tau}, y$ & \\
\hline C-III-e & $\pm i \hat{w}_{1},-\hat{w}_{2}, \hat{w}_{S}$ & $x e^{i \tau}, x e^{-i \tau}, y$ & \\
\hline C-III-f & $\pm i \hat{w}_{1}, i \hat{w}_{2}, \hat{w}_{S}$ & & \\
\hline C-III-g & $\pm i \hat{w}_{1},-i \hat{w}_{2}, \hat{w}_{S}$ & & \\
\hline C-III-h & $\sqrt{3} \hat{w}_{2} e^{i \sigma_{2}}, \pm \hat{w}_{2} e^{i \sigma_{2}}, \hat{w}_{S}$ & $x e^{i \tau}, y, y, \quad y, x e^{i \tau}, y$ & $\checkmark$ \\
\hline C-III-i & $\begin{aligned} & \sqrt{\frac{3\left(1+\tan ^{2} \sigma_{1}\right)}{1+9 \tan \sigma_{1}}} \hat{w}_{2} e^{i \sigma_{1}} \\
& \pm \hat{w}_{2} e^{-i \arctan \left(3 \tan \sigma_{1}\right)}, \hat{w}_{S}\end{aligned}$ & $\begin{array}{l}x, y e^{i \tau}, y e^{-i \tau} \\
y e^{i \tau}, x, y e^{-i \tau}\end{array}$ & \\
\hline$\overline{C \text { C-IV-a* }}$ & $\hat{w}_{1} e^{i \sigma_{1}}, 0, \hat{w}_{S}$ & & \\
\hline C-IV-b & $\hat{w}_{1}, \pm i \hat{w}_{2}, \hat{w}_{S}$ & & \\
\hline C-IV-c & $\begin{array}{c}\sqrt{1+2 \cos ^{2} \sigma_{2}} \hat{w}_{2} \\
\hat{w}_{2} e^{i \sigma_{2}}, \hat{w}_{S}\end{array}$ & & $\checkmark$ \\
\hline C-IV-d* & $\hat{w}_{1} e^{i \sigma_{1}}, \pm \hat{w}_{2} e^{i \sigma_{1}}, \hat{w}_{S}$ & & \\
\hline C-IV-e & $\begin{array}{c}\sqrt{-\frac{\sin 2 \sigma_{2}}{\sin 2 \sigma_{1}}} \hat{w}_{2} e^{i \sigma_{1}} \\
\hat{w}_{2} e^{i \sigma_{2}}, \hat{w}_{S}\end{array}$ & & $\checkmark$ \\
\hline C-IV-f & $\begin{array}{c}\sqrt{2+\frac{\cos \left(\sigma_{1}-2 \sigma_{2}\right)}{\cos \sigma_{1}}} \hat{w}_{2} e^{i \sigma_{1}} \\
\hat{w}_{2} e^{i \sigma_{2}}, \hat{w}_{S}\end{array}$ & & $\checkmark$ \\
\hline $\mathrm{C}-\mathrm{V}^{*}$ & $\hat{w}_{1} e^{i \sigma_{1}}, \hat{w}_{2} e^{i \sigma_{2}}, \hat{w}_{S}$ & $x e^{i \tau_{1}}, y e^{i \tau_{2}}, z$ & \\
\hline
\end{tabular}

up the middle element (I, III, etc) of the vacuum name given in Table 1 refers to the number of independent conditions.

Many of the complex vacua support spontaneous CP violation. However, the model can also lead to complex vacua that do not violate CP. This is due to the symmetry of the potential, as will be shown by a few examples.

\subsection{Example: C-I-a}

This is a well-known case [22], best discussed in the reducible-representation framework. Under complex conjugation (c. c.), the vacuum undergoes the following transformation:

$$
\left(\rho_{1}, \rho_{2}, \rho_{3}\right)=\left(x, x e^{ \pm 2 \pi i / 3}, x e^{\mp 2 \pi i / 3}\right) \stackrel{\text { c.c. }}{\longrightarrow}\left(x, x e^{\mp 2 \pi i / 3}, x e^{ \pm 2 \pi i / 3}\right) .
$$


The vevs of $\phi_{2}$ and $\phi_{3}$ have been complex conjugated. However, the potential is symmetric under the interchange of $\phi_{2}$ and $\phi_{3}$, and since the moduli are the same, it remains invariant under complex conjugation.

\subsection{Example: C-III-a}

In the RRF, this has the form

$$
\left(\rho_{1}, \rho_{2}, \rho_{3}\right)=\left(y, y, x e^{i \tau}\right) \stackrel{\text { c.c. }}{\longrightarrow}\left(y, y, x e^{-i \tau}\right),
$$

whereas in the IRF it has the form

$$
\left(w_{1}, w_{2}, w_{S}\right)=\left(0, \hat{w}_{2} e^{i \sigma_{2}}, \hat{w}_{S}\right) \stackrel{\text { c.c. }}{\longrightarrow}\left(0, \hat{w}_{2} e^{-i \sigma_{2}}, \hat{w}_{S}\right),
$$

In this case, no symmetry operation can "undo" a complex conjugation, and CP is spontaneously violated.

\subsection{Example: C-III-b}

In the IRF this has the form

$$
\left(w_{1}, w_{2}, w_{S}\right)=\left( \pm i \hat{w}_{1}, 0, \hat{w}_{S}\right) \stackrel{\text { c.c. }}{\longrightarrow}\left(\mp i \hat{w}_{1}, 0, \hat{w}_{S}\right) .
$$

At first sight, it looks like this would lead to CP violation. However, we recall that the potential is invariant under the interchange $h_{1} \rightarrow-h_{1}$, so there is no CP violation. The same conclusion is also easily reached in the RRF, and applies also to C-III-d and C-III-e.

\subsection{Example: C-III-c}

In the IRF this has the form

$$
\left(w_{1}, w_{2}, w_{S}\right)=\left(\hat{w}_{1} e^{i \sigma_{1}}, \hat{w}_{2} e^{i \sigma_{2}}, 0\right) \stackrel{\text { c.c. }}{\longrightarrow}\left(\hat{w}_{1} e^{-i \sigma_{1}}, \hat{w}_{2} e^{-i \sigma_{2}}, 0\right) .
$$

Since $w_{S}=0$, a rephasing allows for the removal of one phase:

$$
\left(w_{1}, w_{2}, w_{S}\right)=\left(\hat{w}_{1} e^{i \sigma}, \hat{w}_{2} e^{-i \sigma}, 0\right) \stackrel{\text { c.c. }}{\longrightarrow}\left(\hat{w}_{1} e^{-i \sigma}, \hat{w}_{2} e^{i \sigma}, 0\right) .
$$

This vacuum requires $\lambda_{4}=0$, in which case the potential is symmetric under the interchange $h_{1} \leftrightarrow$ $h_{2}$. However, in the general case, for $\hat{w}_{1} \neq \hat{w}_{2}$, this vacuum leads to CP violation.

We proposed adding a soft term to avoid massless neutral scalars in this case. In section 9 of our paper [21] we did not introduce the most general soft breaking terms and we incorrectly state that with our choice there is still CP violation.

\subsection{Example: The Pakvasa-Sugawara vacuum}

The following complex vacuum was identified by Pakvasa and Sugawara many years ago [23]:

$$
\left(w_{1}, w_{2}, w_{S}\right)=\left(\hat{w} e^{i \sigma}, \hat{w} e^{-i \sigma}, \hat{w}_{S}\right) .
$$

Superficially, this looks like it might lead to CP violation. However, for consistency, it requires $\lambda_{4}=0$, in which case the potential is symmetric under the interchange $h_{1} \leftrightarrow h_{2}$. Hence, there is no $\mathrm{CP}$ violation. (This vacuum is contained in C-III-f, C-III-g and C-IV-e, depending on which additional conditions are imposed, in addition to $\lambda_{4}=0$.) 


\subsection{Example: The Ivanov-Nishi vacuum}

The following complex vacuum was identified by Ivanov and Nishi [24]:

$$
\left(w_{1}, w_{2}, w_{S}\right)=\left(\hat{w} e^{i \sigma}, \hat{w} e^{i \sigma}, \hat{w}_{S}\right)
$$

Also this one requires $\lambda_{4}=0$. Additional conditions on this solution imply that it does not lead to $\mathrm{CP}$ violation. In fact, it is a special case of C-III-f, C-III-g or C-IV-d, depending on which additional conditions are imposed, together with $\lambda_{4}=0$.

Note that the vacuum

$$
\left(\rho_{1}, \rho_{2}, \rho_{3}\right)=x\left(e^{i \tau}, e^{i \tau}, 1\right)
$$

which is a special case of C-III-a (with $y=x$ ), obtained after a complex conjugation and an overall phase rotation by $e^{i \tau}$, violates $\mathrm{CP}$, whereas

$$
\left(w_{1}, w_{2}, w_{S}\right)=\hat{w}\left(e^{i \sigma}, e^{i \sigma}, 1\right)
$$

does not. It is just a special case of the Ivanov-Nishi vacuum. While these two vacua, Eqs. (3.10) and (3.11) have the same form, the important difference, which leads to opposite conclusions about $\mathrm{CP}$ violation, is the fact that they refer to different frameworks. The two frameworks represent different symmetries among the three fields.

A detailed discussion of all the vacua of Table 1 is given in Ref. [21].

\section{Concluding remarks}

We have discussed two important features of multi-Higgs models. These are: the fact that such models may provide new sources of $\mathrm{CP}$ violation as well as good dark matter candidates. Sources of CP violation beyond the SM are required to explain the observed baryon asymmetry of the Universe and their effects may be observed soon at the LHC or in future colliders. New sources of $\mathrm{CP}$ violation may manifest themselves both in the scalar, the gauge and in the flavour sectors. The recently discovered Higgs boson at the LHC has been under intense experimental study and it looks as if it may closely behave as a standard-like Higgs boson. However, on one hand, there is still room for deviations from SM couplings for the discovered boson and on the other hand, these models predict additional scalars, which may soon be discovered. At present, there is a hint for a new $750 \mathrm{GeV}$ boson both from ATLAS and CMS [25]. The nature of dark matter is another puzzle constituting one of the most important open questions in our field.

Extensions of the scalar sector allow for a large number of new parameters. Symmetries play the rôle of reducing this number and at the same time of establishing connections among different phenomena. We have seen that the $S_{3}$ symmetric potential has a very rich structure. Some of these vacua require $\lambda_{4}=0$ for consistency reasons. In this case the potential acquires an additional $\mathrm{SO}(2)$ symmetry as mentioned in section 3. Spontaneous breaking of this continuous symmetry would then lead to scalar massless states which are experimentally ruled out. One possible way out is to include soft terms in the Higgs potential breaking this symmetry. Soft breaking terms may also 
have interesting implications for spontaneous CP violation as pointed out long ago [26]. Some of the vacua listed in Table 1 have vanishing vevs for some fields. When endowed with a stabilizing symmetry, like $Z_{2}$, for example, those fields might represent dark matter.

Acknowledgements: We thank the local organizers of Corfu 2015 for the very fruitful scientific meeting and the warm hospitality. The work of DE-C and MNR was partially supported by Fundação para a Ciência e a Tecnologia (FCT, Portugal) through the projects CERN/FIS-NUC/0010/2015, and CFTP-FCT Unit 777 (UID/FIS/00777/2013) which are partially funded through POCTI (FEDER), COMPETE, QREN and EU. The work of PO was supported in part by the Research Council of Norway.

\section{References}

[1] A. Riotto and M. Trodden, Ann. Rev. Nucl. Part. Sci. 49 (1999) 35 doi:10.1146/annurev.nucl.49.1.35 [hep-ph/9901362].

[2] T. D. Lee, Phys. Rev. D 8 (1973) 1226. doi:10.1103/PhysRevD.8.1226

[3] E. Accomando et al., hep-ph/0608079.

[4] B. Grzadkowski, O. M. Ogreid and P. Osland, JHEP 1401 (2014) 105 doi:10.1007/JHEP01(2014)105 [arXiv:1309.6229 [hep-ph]].

[5] L. Basso, A. Lipniacka, F. Mahmoudi, S. Moretti, P. Osland, G. M. Pruna and M. Purmohammadi, JHEP 1211 (2012) 011 doi:10.1007/JHEP11(2012)011 [arXiv:1205.6569 [hep-ph]].

[6] L. Basso, A. Lipniacka, F. Mahmoudi, S. Moretti, P. Osland, G. M. Pruna and M. Purmohammadi, PoS Corfu 2012 (2013) 029 [arXiv:1305.3219 [hep-ph]].

[7] D. Fontes, J. C. Romão, R. Santos and J. P. Silva, JHEP 1506 (2015) 060 doi:10.1007/JHEP06(2015)060 [arXiv:1502.01720 [hep-ph]].

[8] D. Fontes, J. C. Romão, R. Santos and J. P. Silva, Phys. Rev. D 92 (2015) 5, 055014 doi:10.1103/PhysRevD.92.055014 [arXiv:1506.06755 [hep-ph]].

[9] L. Lavoura and J. P. Silva, Phys. Rev. D 50 (1994) 4619 doi:10.1103/PhysRevD.50.4619 [hep-ph/9404276].

[10] F. J. Botella and J. P. Silva, Phys. Rev. D 51 (1995) 3870 doi:10.1103/PhysRevD.51.3870 [hep-ph/9411288].

[11] B. Grzadkowski, O. M. Ogreid and P. Osland, JHEP 1411 (2014) 084 doi:10.1007/JHEP11(2014)084 [arXiv:1409.7265 [hep-ph]].

[12] G. C. Branco, M. N. Rebelo and J. I. Silva-Marcos, Phys. Lett. B 614 (2005) 187 doi:10.1016/j.physletb.2005.03.075 [hep-ph/0502118].

[13] J. F. Gunion and H. E. Haber, Phys. Rev. D 72 (2005) 095002 doi:10.1103/PhysRevD.72.095002 [hep-ph/0506227].

[14] D. Chang, W. Y. Keung and I. Phillips, Phys. Rev. D 48 (1993) 4045 doi:10.1103/PhysRevD.48.4045 [hep-ph/9307232].

[15] D. Chang, W. Y. Keung and P. B. Pal, Phys. Rev. D 51 (1995) 1326 doi:10.1103/PhysRevD.51.1326 [hep-ph/9407294]. 
[16] B. Grzadkowski, O. M. Ogreid and P. Osland, arXiv:1603.01388 [hep-ph].

[17] J. F. Gunion and H. E. Haber, Phys. Rev. D 67 (2003) 075019 doi:10.1103/PhysRevD.67.075019 [hep-ph/0207010].

[18] B. Grzadkowski, O. M. Ogreid and P. Osland, PoS CORFU 2014 (2015) 086 [arXiv:1504.06076 [hep-ph]].

[19] D. Das and U. K. Dey, Phys. Rev. D 89 (2014) no.9, 095025 Erratum: [Phys. Rev. D 91 (2015) no.3, 039905] doi:10.1103/PhysRevD.91.039905, 10.1103/PhysRevD.89.095025 [arXiv:1404.2491 [hep-ph]].

[20] E. Derman, Phys. Rev. D 19 (1979) 317. doi:10.1103/PhysRevD.19.317

[21] D. Emmanuel-Costa, O. M. Ogreid, P. Osland and M. N. Rebelo, JHEP 1602 (2016) 154 doi:10.1007/JHEP02(2016)154 [arXiv:1601.04654 [hep-ph]].

[22] G. C. Branco, J. M. Gerard and W. Grimus, Phys. Lett. B 136 (1984) 383. doi:10.1016/0370-2693(84)92024-0

[23] S. Pakvasa and H. Sugawara, Phys. Lett. B 73 (1978) 61. doi:10.1016/0370-2693(78)90172-7

[24] I. P. Ivanov and C. C. Nishi, JHEP 1501 (2015) 021 doi:10.1007/JHEP01(2015)021 [arXiv:1410.6139 [hep-ph]].

[25] J. Olsen, CMS physics results from Run 2 presented on Dec. 15th, 2015, M. Kado, ATLAS physics results from Run 2 presented on Dec. 15th, 2015, https://indico.cern.ch/event/442432/. ATLAS Collaboration, ATLAS-CONF-2015-081, https://atlas.web.cern.ch/ Atlas/GROUPS/PHYSICS/CONFNOTES/ATLAS-CONF-2015-081/. CMS Collaboration, CMS PAS EXO-15-004, https://cds.cern.ch/record/ 2114808/files/EXO-15-004-pas.pdf.

[26] G. C. Branco and M. N. Rebelo, Phys. Lett. B 160 (1985) 117. doi:10.1016/0370-2693(85)91476-5 